Check for updates

London

Cite this as: $B M J$ 2021;373:n1445 http://dx.doi.org/10.1136/bmi.n1445 Published: 04 June 2021

\section{Covid-19: Delta variant is now UK's most dominant strain and spreading through schools}

\author{
Ingrid Torjesen
}

The delta covid-19 variant (B.1.617.2), which was first detected in India, has overtaken the alpha variant, better known as the UK or Kent variant (B.1.1.7), to make it the most dominant strain circulating in the UK, Public Health England has said.

This is a concern because there is evidence that the risk of hospital admission is higher in people with the delta variant, it said. Data also indicate that the variant is spreading rapidly through England's schools.

Public Health England's latest weekly coronavirus data on circulating variants show there were 5472 new cases of the delta variant in the UK in the week to 26 May, bringing the total number of cases of the variant detected to $12431 .^{1}$

In the week to 2 June a total of 278 people with the delta variant attended hospital emergency departments, and 94 people were admitted to hospital overnight. The previous week 201 people with the variant attended A\&E and 43 were admitted. Most of those admitted had not been vaccinated.

Public Health England confirmed that its data showed an increase in outbreaks and clusters involving variants at primary and secondary schools in recent weeks, though it has not published these data in detail. In the most recent four week period there were 97 confirmed covid-19 outbreaks in primary and secondary schools that were linked to at least one variant of concern, equates to around one outbreak at every 250 schools, it said.

Teaching unions and advocacy groups have written to Public Health England demanding more information on outbreaks in schools.

A letter on 26 May from eight trade unions representing schools and colleges said, "There are growing concerns around the variant B.1.617.2 and reports from areas such as Bolton that cases are growing fastest amongst school age children, with cases in Bolton higher now than at any point during the pandemic."2

In its reply on 4 June to a similar letter from the advocacy group Citizens, Public Health England said that it needed "more time" to provide detailed data.

Deepti Gurdasani, a senior lecturer in machine learning at Queen Mary University and a spokesperson for Citizens, said, "The limited data released from PHE clearly shows that outbreaks of the delta variant in schools exceed those in any other defined setting and that cases among secondary school children are the highest across all age groups, and rising rapidly."
Latest data from the Office for National Statistics for the week to 29 May show that numbers of cases of covid-19 have been rising fastest in schoolchildren in years 7 to $11 .^{3}$ Data from Public Health England show that the number of outbreaks involving variants managed by health protection teams in educational settings have been rising for some weeks and trebled in the last two weeks of May. ${ }^{4}$ In the week to 1 June health protection teams managed more outbreaks in educational settings than in any other defined setting, and the vast majority of outbreaks involved the delta variant.

Gurdasani said, "It's clear that our government's policies are putting children and communities at risk. We urgently need transparency on these data to review current policies in schools, given the role of school transmission in the rapid spread of this new variant across England."

Paul Whiteman, general secretary of the National Association of Head Teachers, said that the data on the spread of the delta variant in schools were a matter of concern and raised serious questions about the government's decision to remove some of the mitigation measures in schools last month, including dropping the requirement to wear masks from 17 May.

"We have been hearing from our members that more and more schools are having to close multiple classes or 'bubbles,' particularly in areas with higher case numbers. This latest official data release appears to support those concerns," he said.

1 Public Health England. Covid-19 variants: genomically confirmed case numbers. 3 Jun 2021. https://www.gov.uk/government/publications/covid19-variants-genomically-confirmed-case-numbers.

2 National Association of Head Teachers. Government data on covid variant cases linked to schools should be published "immediately," say education unions. 27 May 2021. https://www.naht.org.uk/news-and-opinion/pressroom/government-data-on-covid-variant-cases-linked-to-schools-shouldbe-published-immediately-say-education-unions.

3 Office for National Statistics. Coronavirus (covid-19) infection survey, UK. 4 Jun 2021. https://www.ons.gov.uk/peoplepopulationandcommunity/healthandsocialcare/conditionsanddiseases/bulletins/coronaviruscovid19infectionsurveypilot/4june2021\#age-analysis-of-the-number-ofpeople-who-had-covid-19.

4 Public Health England. SARS-CoV-2 variants of concern and variants under investigation: technical briefing 14. 3 Jun 2021. https://assets.publishing.service.gov.uk/government/uploads/system/uploads/attachment_data/file/991343/Variants_of_Concern_VOC_Technical_Briefing_14.pdf.

This article is made freely available for use in accordance with BMJ's website terms and conditions for the duration of the covid-19 pandemic or until otherwise determined by BMJ. You may use, download and print the article for any lawful, non-commercial purpose (including text and data mining) provided that all copyright notices and trade marks are retained. 